Article

\title{
Vortex Structure and Kinematics of Encased Axial Turbomachines ${ }^{\dagger}$
}

\author{
Peter F. Pelz *, Paul Taubert and Ferdinand-J. Cloos
}

Chair of Fluid Systems, Technische Universität Darmstadt, Otto-Berndt-Str. 2, 64287 Darmstadt, Germany; paul.taubert@fst.tu-darmstadt.de (P.T.); ferdinand.cloos@fst.tu-darmstadt.de (F.-J.C.)

* Correspondence: peter.pelz@fst.tu-darmstadt.de; Tel.: +49-6151-16-27100

+ This paper is an extended version of our paper published in Proceedings of the 17th International Symposium on Transport Phenomena and Dynamics of Rotating Machinery (ISROMAC 2017).

Received: 26 January 2018; Accepted: 23 April 2018; Published: 27 April 2018

check for updates

\begin{abstract}
This paper models the kinematics of the vortex system of an encased axial turbomachine at part load and overload applying analytical methods. Thus far, the influence of the casing and the tip clearance on the kinematics have been solved separately. The vortex system is composed of a hub, bound and tip vortices. For the nominal operating point $\varphi \approx \varphi_{\text {opt }}$ and negligible induction, the tip vortices transform into a screw. For part load operation $\varphi \rightarrow 0$ the tip vortices wind up to a vortex ring, i.e., the pitch of the screw vanishes. The vortex ring itself is generated by bound vortices rotating at the angular frequency $\Omega$. The hub vortex induces a velocity on the vortex ring causing a rotation at the sub-synchronous frequency $\Omega_{\text {ind }}=0.5 \Omega$. Besides, the vortex ring itself induces an axial velocity. Superimposed with the axial main flow this results in a stagnation point at the tube wall. This stagnation point may wrongly be interpreted as dynamic induced wall stall. For overload operation $\varphi \rightarrow \infty$ the vortex system of the turbomachine forms a horseshoe, i.e., the pitch of the screw becomes infinite. Both hub and tip vortices are semi-infinite, straight vortex filaments. The tip vortices rotate against the rotating direction of the turbomachine due to the induction of the hub vortex yielding the induced frequency $\Omega_{\text {ind }}=-0.5 \Omega / s$ with the tip clearance $s$.
\end{abstract}

Keywords: vortex dynamics; potential flow; part load; overload; sub-synchronous frequency; kinemtatic induced noise

\section{Introduction and Literature Review}

By now, the common understanding is that rotating stall as well as the resulting noise and vibration within a turbomachine is a dynamic effect. This means that frictional forces lead to boundary layer separation and eventually stall in rotating machines [1,2]. This understanding is recently confirmed by Cloos et al. [3] both experimentally and analytically for the most generic machine, a flow through a coaxial rotating circular tube. According to Cloos et al. [3], wall stall-a term coined by Greitzer [1] in contrast to blade stall —is caused at part load by the interaction of axial boundary layer and swirl boundary layer flow, i.e., the influence of centrifugal force on axial momentum. For wall stall the axial velocity component $u_{z}$ vanishes at the line $z=-z_{0}, r=a$ (axial coordinate in mean flow direction $z$, distance $z_{0}$ from the reference point on the line of symmetry $r=0$, radial coordinate $r$ and tube radius $a$; cf. Figure 1 ).

This paper analyzes the flow situation in encased axial turbomachines for small viscous friction. This work shows that wall stall, i.e., $u_{z}\left(-z_{0}, a\right)=0$ can also be a result of kinematics only due to induced velocities of the vortex system superimposed with the axial main flow. The structure of the vortex system, especially the tip vortices, depends on the operating point of the turbomachine. Furthermore, the tip vortices rotate with a sub-synchronous frequency $\Omega_{\text {ind }}$ (Karstadt et al. [4] and 
$\mathrm{Zhu}$ [5]). The aim of the present paper is to analyze the influence of the vortex system in encased axial turbomachines and its circulation strength on the observed phenomena yielding the research questions:

1. Is it possible to explain by means of analytical methods the sub-synchronous frequencies observed for turbomachines?

2. Can wall stall be a result of kinematics only?

To answer these questions, this paper first employs vortex theory for an encased axial turbomachine followed by the application of fundamental solutions. For machines without casing such as wind turbines and screw propellers, vortex theory is well described by Betz [6], Goldstein [7], Glauert [8] and van Kuik [9]. The method is not yet developed in such a degree for encased turbomachines requiring the flow potential of a vortex ring inside a tube.

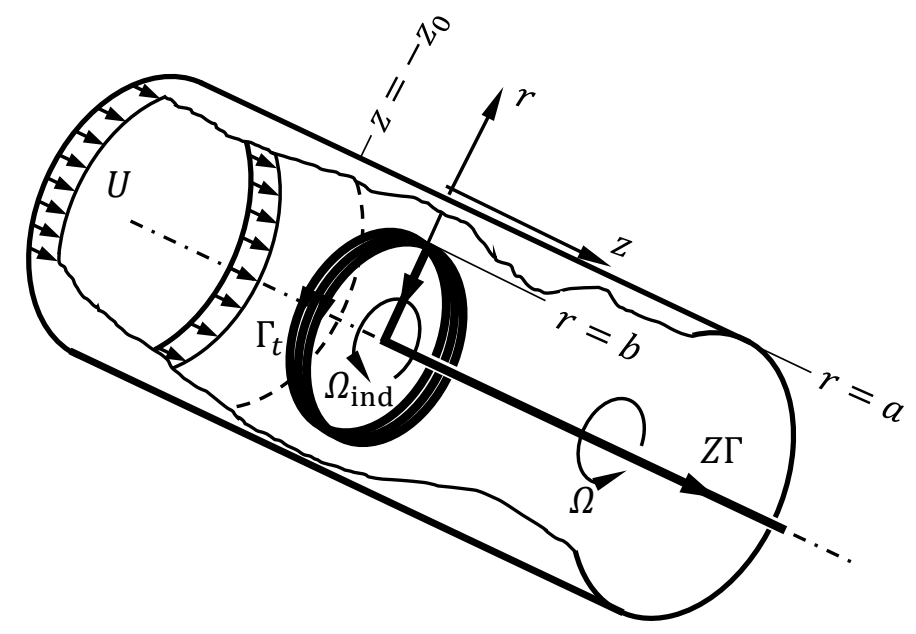

Figure 1. A coaxial vortex ring of transient strength $\Gamma_{t}$ and radius $b$ in a circular tube of radius $a$, according to the case $\varphi \rightarrow 0$. The sketch is for $Z=1$, i.e., one bound vortex only to improve clarity.

In addition, to enlarge the investigation on the whole operating range of a turbomachine, we investigate the structure and kinematics of the vortex system at heavy overload applying theory of functions (complex analysis). For turbomachines, the flow number $\varphi:=U / \Omega a$ defines the operating point (e.g., part load or overload). The flow number is the ratio of axial free-stream velocity $U$ to the circumferential velocity $\Omega a$ where $\Omega=2 \pi n$ is the rotational speed (the scaling to $\Omega a$ and not to $\Omega b$ with the blade tip radius $b$ is common in the context of turbomachines and therefore used here as well [10]).

For the nominal operating point $\varphi \approx \varphi_{\text {opt }}$ and negligible induction, the vortex system of an encased axial turbomachine consists of a hub, $Z$ bound and $Z$ tip vortices, with $Z$ the number of blades. The tip vortices transform into helices with a pitch of $2 \pi \varphi a$.

For part load operation $\varphi \rightarrow 0$ (see Figure 1), the $Z$ helices "roll up" and form a vortex ring, i.e., the pitch of the helices vanishes. The vortex ring is continuously generated by the bound vortex system. Hence, the coaxial vortex ring strength is transient. A nice picture for this vortex ring is that of a thread spool rolling up and gaining strength over time. This picture explains some transient phenomena using kinematic arguments only.

The case of heavy overload occurs for infinitely high flow numbers $\varphi \rightarrow \infty$ (see Figure 2). The hub, the bound and the tip vortices form a horseshoe, i.e., the pitch of the helices becomes infinite. Both hub and tip vortices are semi-infinite, straight vortex filaments. In real turbomachines, the flow number cannot be adjusted to infinity but is limited to a maximum value $\hat{\varphi}$ due to flow rate limitations and geometric restrictions. Nevertheless, the analysis of this limiting case is important for the basic understanding of the vortex system in axial turbomachines. 
Investigations of vortex systems in fluid dynamics trace back to the work of Helmholtz [11] who formulated the Helmholtz's theorems as a basis for the research concerning rotational fluid motion.

Didden [12] performed measurements of the rolling-up process of vortex rings and compared the results with similarity laws for the rolling-up of vortex sheets.

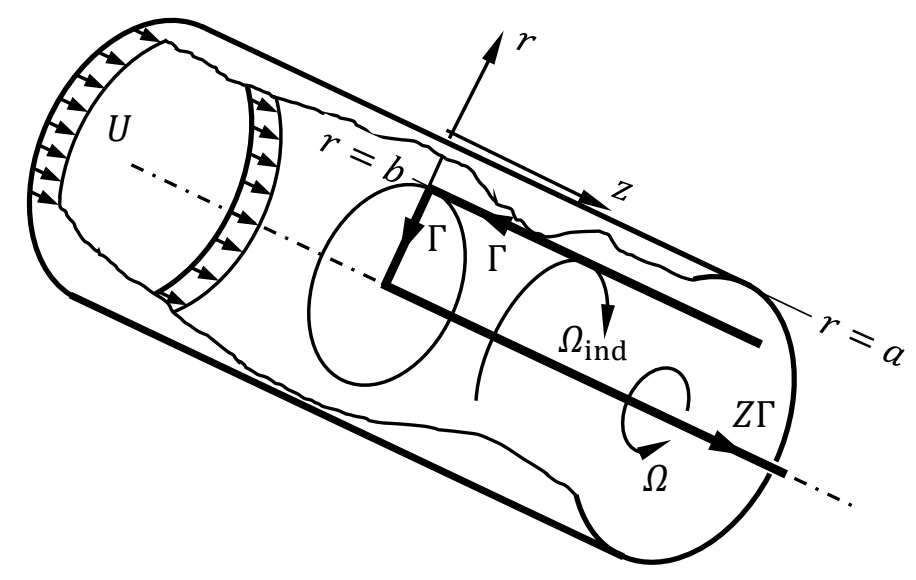

Figure 2. A horseshoe vortex of strength $\Gamma$ and radius $b$ in a circular tube of radius $a$, according to the case $\varphi \rightarrow \infty$. The sketch is for $Z=1$, i.e., one bound vortex only to improve clarity.

Besides the investigation of vortex kinematics, a broad research field on vortex structures in turbomachines is the experimental and numerical analysis of acoustic and noise emission of tip vortices [13-16]. The noise of a fan is noticeable by a CPU, car or a rail vehicle cooler. All three examples are met in everyday life. One of the main reasons for the noise is the gap $s:=(a-b) / a$ between the housing and the impeller tip. With increasing gap the noise emission and the energy dissipation increase [17,18]. Karstadt et al. [19] and Zhu [5] investigated noise and dissipation due to tip vortices.

The kinematics for an encased axial turbomachine operating at part load or overload are not fully understood yet. Especially the basic kinematics of these phenomena are not sufficiently analyzed.

To develop physical understanding of the whole picture in detail, the paper is organized as follows. Section 2 uses vortex theory to determine the strength of the vortices. Subsequently, Section 3 derives the velocity potential of a coaxial vortex ring within a circular tube at part load and the induced rotating frequency. The flow potential and the induction at overload is introduced in Section 4. The paper closes with a short outlook to potential applications in Section 5 and a discussion in Section 6.

\section{Vortex Theory}

An encased axial turbomachine with $Z$ impellers is considered. The sketched vortex system (see Figures 1 and 2) results from the $Z$ bound vortices. The generation of a bound vortex was explained by Prandtl [20] using arguments of boundary layer theory and Kelvin's circulation theorem. The presence of viscosity is essential for the creation of the bound vortex but the generation phase is not in the scope of this paper. For vortex generation, we would like to refer the reader to the work of Prandtl [20].

By vortex theory, each blade $1, \ldots, Z$ of length $b$ is represented by its bound vortex of strength $\Gamma$. For simplicity, this investigation assumes $\Gamma$ to be constant in radial direction along the blade from $r=0$ to $r=b$. As a vortex filament cannot end in a fluid due to Helmholtz's vortex theorem, a free, trailing vortex springs at each blade end $r=0$ and $r=b$ (see Figures 1 and 2). These vortices are of the same strength as the bound vortex. At the inner end $r=0$, a straight semi-infinite vortex line $0 \leq z<\infty$ of strength $Z \Gamma$ - the so-called hub vortex-attaches to the blade. The tip vortices at the outer end $r=b$ are helices. The axial distance of the each helix winding, i.e., the helix pitch, is given 
by $U / n=2 \pi a \varphi$. Depending on the load, these helices either "wind up" $(\varphi \rightarrow 0)$ forming a vortex ring or stretch to infinity $(\varphi \rightarrow \infty)$ yielding a straight, semi-infinite vortex line.

Regardless of the flow number $\varphi$, the semi-infinite straight vortex line at $r=0$ induces the circumferential velocity $Z \Gamma /(4 \pi b)$ at $z=0, r=b$ due to the Biot-Savart law. Hence, the induced rotational speed is $\Omega_{\text {ind }}=Z \Gamma /\left(4 \pi b^{2}\right)$.

In a next step, this analysis calculates the vortex strength $Z \Gamma$ employing the angular momentum equation and the energy equation. On the one hand, the axial component of the angular momentum equation is $Z \Gamma / 2 \pi=\mathrm{d} M / \mathrm{d} \dot{m}$. Here, $M$ is the axial torque component applied by the turbomachine to the fluid and $\dot{m}$ the mass flux. A more detailed explanation is given in Appendix A. Multiplying the momentum equation by $\Omega$ yields $Z \Gamma n=\mathrm{d} P / \mathrm{d} m . P=M \Omega$ is the power applied to the fluid by means of the rotating bound vortices. On the other hand, the energy equation for an adiabatic flow reads $\mathrm{d} P / \mathrm{d} \dot{m}=\Delta h_{t}$, with $\Delta h_{t}$ being the difference in total enthalpy experienced by a fluid particle passing the cross-section $z=0$. Both arguments result in the relation $Z \Gamma n=\Delta h_{t}$.

From turbomachine theory, the expression $\Delta h_{t}=(\Omega b)^{2}(1-\varphi / \hat{\varphi})$ can be derived from the equation mentioned above. The dimensionless design parameter $\hat{\varphi}$ equals the tangent of the blade's trailing edge angle $\beta_{2}$, i.e., $\hat{\varphi}=\tan \beta_{2}$. Hence, the relation between $Z \Gamma$ and $\Omega$ yields

$$
\frac{Z \Gamma n}{(\Omega b)^{2}}=1-\frac{\varphi}{\hat{\varphi}} .
$$

As Equation (1) shows, the total change in circulation $Z \Gamma$ along the plane of the machine is linked to the flow number $\varphi$ by Euler's turbine equation.

\section{The Vortex System at Heavy Part Load}

For the limiting case of interest $\varphi \rightarrow 0$, the relation between $Z \Gamma$ and $\Omega$, Equation (1), yields $\Delta h_{t}=Z \Gamma n=(\Omega b)^{2}$. This results in an induced sub-synchronous frequency

$$
\frac{\Omega_{\text {ind }}}{\Omega}=\frac{1}{2}, \quad \text { for } \varphi \rightarrow 0 .
$$

Here, the calculation neglects the self-induction of the vortex ring. This idealization is to some extent inconsistent but allows obtaining a simple solution for the kinematics of an encased axial turbomachine.

The induced frequency, Equation (2), is in surprisingly good agreement with measured sub-synchronous frequencies $0.5 \Omega-0.7 \Omega$ of rotating stall of compressors, fans and pumps at part load operation [10] and may result in a rethinking of rotating stall from a kinematic perspective.

This investigation is now set to analyze the kinematics of coaxial vortex rings of radius $b$ and maximal strength $\Gamma_{t}=Z \Gamma n t<(\Omega b)^{2} t$, with time $t$, as sketched in Figure 1. By doing so, Laplace's equation

$$
\frac{1}{r} \frac{\partial}{\partial r}\left(r \frac{\partial \Phi}{\partial r}\right)+\frac{\partial^{2} \Phi}{\partial z^{2}}=0
$$

for the velocity potential $\Phi$, with $\vec{u}=\nabla \Phi$, is solved for an incompressible, axisymmetric flow. The velocity potential for a coaxial ring filament in a circular tube yields

$$
\phi(r, z):=\frac{\Phi}{U a}=\frac{z}{a}-2 \tau \beta^{2} \sum_{n=1}^{\infty} \frac{J_{1}\left(k_{n} \beta\right)}{k_{n} J_{0}^{2}\left(k_{n}\right)} J_{0}\left(k_{n} \frac{r}{a}\right) \exp \left(-k_{n} \frac{|z|}{a}\right)
$$

with $J_{0}, J_{1}$ the Bessel function of orders 0 and 1 , respectively, and $k_{n}$ the zeros $n=1, \ldots, \infty$ of the function $J_{0}^{\prime}\left(k_{n}\right)=-J_{1}\left(k_{n}\right)=0$. Appendix B gives a more detailed derivation for this result. The dimensionless velocity potential $\phi$ depends on the dimensionless ring radius $\beta:=b / a$ and the dimensionless vortex strength $\tau:=\Gamma_{t} / 2 b U$. Since $\Gamma_{t}$ increases linearly in time, $\tau$ can also be interpreted as a parametric time of the process. 
Stokes stream function for this flow is (using the integrability conditions $\partial \psi / \partial z=-r \partial \phi / \partial r$ and $\partial \psi / \partial r=r \partial \phi / \partial z)$

$$
\psi(r, z):=\frac{\Psi}{U a^{2}}=-\frac{1}{2}\left(1-\frac{r^{2}}{a^{2}}\right)+2 \tau \beta^{2} \sum_{n=1}^{\infty} \frac{J_{1}\left(k_{n} \beta\right)}{k_{n} J_{0}^{2}\left(k_{n}\right)} J_{1}\left(k_{n} \frac{r}{a}\right) \frac{r}{a} \exp \left(-k_{n} \frac{|z|}{a}\right) .
$$

With the stream function, the radial velocity component

$$
\frac{u_{r}(r, z)}{U}=2 \tau \beta^{2} \sum_{n=1}^{\infty} \frac{J_{1}\left(k_{n} \beta\right)}{J_{0}^{2}\left(k_{n}\right)} J_{1}\left(k_{n} \frac{r}{a}\right) \exp \left(-k_{n} \frac{|z|}{a}\right)
$$

and the axial velocity component

$$
\frac{u_{z}(r, z)}{U}=1+2 \tau \beta^{2} \sum_{n=1}^{\infty} \frac{J_{1}\left(k_{n} \beta\right)}{J_{0}^{2}\left(k_{n}\right)} J_{0}\left(k_{n} \frac{r}{a}\right) \exp \left(-k_{n} \frac{|z|}{a}\right)
$$

are given. The velocity field (Equations (6) and (7)) takes the induction of the vortex ring into account. At the stagnation point $z= \pm z_{0}$, the axial velocity $u_{z}$ vanishes for

$$
1=-2 \tau \beta^{2} \sum_{n=1}^{\infty} \frac{J_{1}\left(k_{n} \beta\right)}{J_{0}^{2}\left(k_{n}\right)} J_{0}\left(k_{n}\right) \exp \left(-k_{n} \frac{\left|z_{0}\right|}{a}\right)
$$

Equation (8) is implicit for the stagnation point $z_{0}=z_{0}(\tau, \beta)$. Figure 3 shows Stokes stream function and the stagnation points at $z= \pm z_{0}$ for different circulation strengths $\tau$ of the vortex for $\beta=0.8$. With increasing $\tau$, the stagnation point $z_{0}$ moves away from the plane of the turbomachine $z=0$. This is because the induced velocity of the vortex ring increases with respect to the free-stream velocity. Hence, the limiting case $\tau \rightarrow 0$ represents an undisturbed flow through a rigid pipe without vortices. For the limiting case $\tau \rightarrow \infty$, the free-stream velocity disappears and only the two vortices at $r= \pm b, z=0$ would be visible.

a) $\tau=0.8$

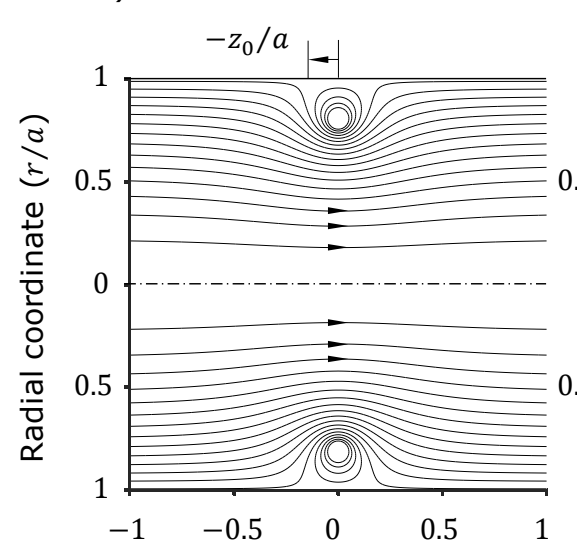

b) $\tau=2.5$

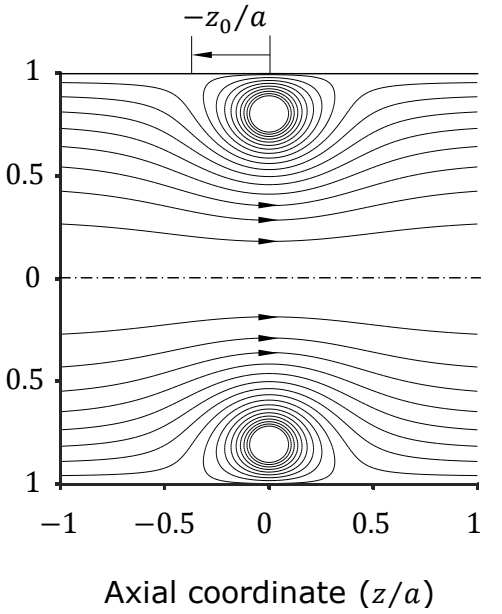

c) $\tau=5.0$

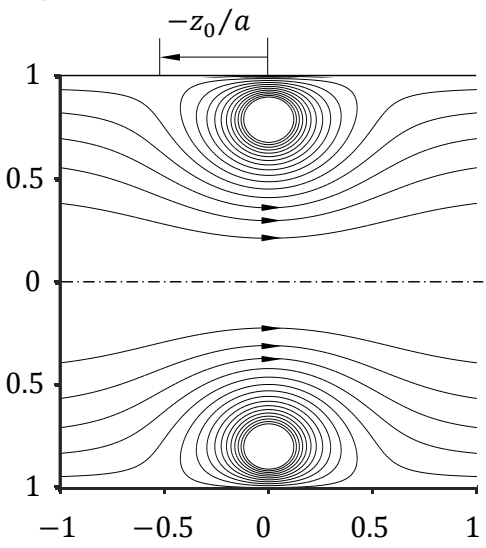

Figure 3. Contour plots of the stream function (Equation (5)) for a vortex ring with the strength: (a) $\tau=0.8$; (b) $\tau=2.5$; and (c) $\tau=5.0$, for $\beta=0.8$.

\section{The Vortex System at Heavy Overload}

Figure 4 visualizes the vortex system of an axial turbomachine composed of $Z=1$ impeller blade at heavy overload $\varphi \rightarrow \infty$ ( $Z=1$ is chosen to improve clarity only). For this limiting case, the flow at cross section A-A far downstream of the machine is a plane potential flow. It can be described using theory of functions (complex analysis) [21]. Mirrored tip vortices are necessary to fulfill the 
kinematic boundary condition on the tube wall. These mirrored vortices are located in the housing and on the rotational axis of the turbomachine. Considering the tip vortex and its mirrored conjugates only, i.e., neglecting the hub vortex as a first step, one obtains the system visualized in Figure 4, bottom left. The vortex on the axis and the hub vortex feature identical magnitude but opposing rotating directions. Adding the hub vortex yielding the complete system hence results in the annulation of these two vortices (see Figure 4, bottom right).
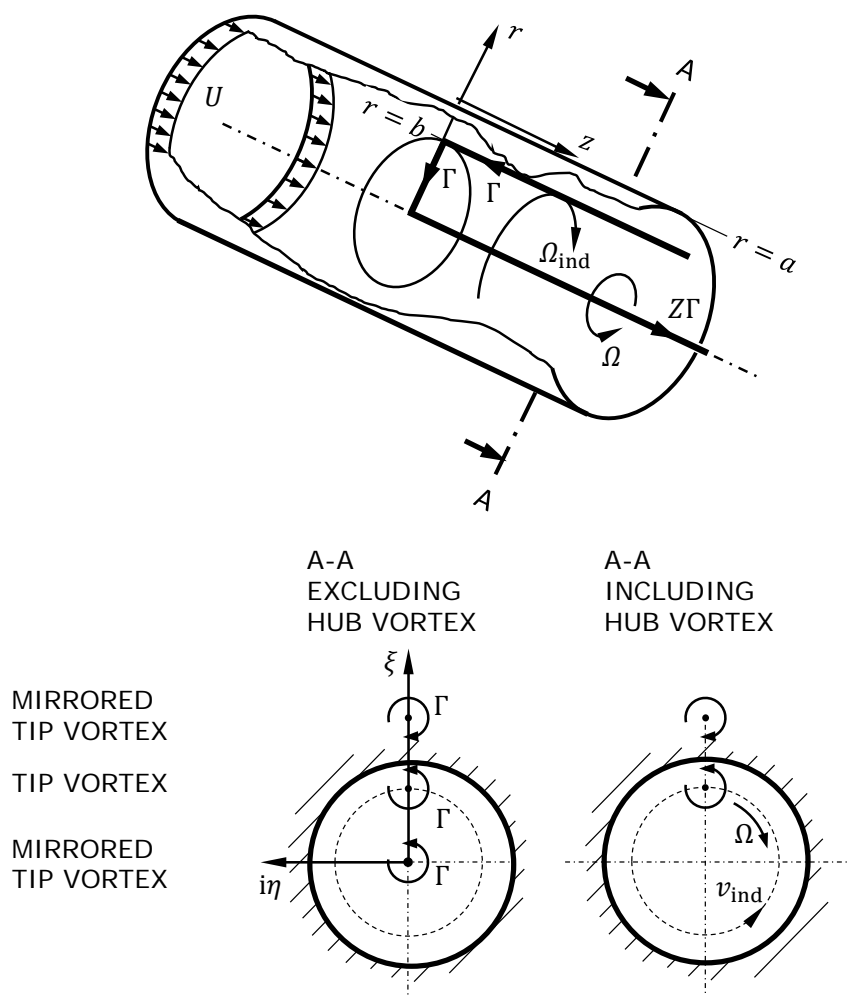

Figure 4. The vortex system of an axial turbomachine at overload, $Z=1$.

In the following notation, the complex coordinates $\zeta=\xi+\mathrm{i} \eta=r \exp (\mathrm{i} \theta)$ are composed of a real part $\xi=r \cos \theta$ and an imaginary part $\eta=r \sin \theta$, with $i=\sqrt{-1}$. The complex potential $F(\xi, \eta)$ is divided into the real part, which is the velocity potential $\Re[F(\xi, \eta)]=\Phi(\xi, \eta)$ and the imaginary part which is the stream function $\Im[F(\xi, \eta)]=\Psi(\xi, \eta) . \bar{F}$ is the complex conjugate of $F$.

For the considered potential flow, the tip vortex at radial position $b=(1-s) a$ yields the complex potential

$$
F_{1}(\zeta)=-\frac{i \Gamma}{2 \pi} \ln (\zeta-b)
$$

Here, $s$ is the dimensionless gap. The Milne-Thomson circle theorem [22] is applied to derive the complex potential satisfying the kinematic boundary condition at the wall. This theorem postulates a resulting complex potential

$$
F_{2}(\zeta)=F_{1}(\zeta)+\bar{F}_{1}\left(\frac{a^{2}}{\zeta}\right)
$$

for a potential $F_{1}$ and the mirrored potential at the surrounding wall. Adding the potential of the mirrored tip vortex on the axis of the turbomachine $\zeta=0$ (see Figure 4 , bottom right), yields for the complex flow potential

$$
F^{\prime}(\zeta)=-\frac{\mathrm{i} \Gamma}{2 \pi}\left[\ln (\zeta-b)-\ln \left(\zeta-\frac{a^{2}}{b}\right)+\ln \zeta\right]+\text { const. }
$$


The tip vortex at $\zeta=b$ with the circulation $\Gamma$ necessitates a mirrored vortex at $\zeta=0$ with the same magnitude of circulation and a mirrored vortex in the housing at $\zeta=a^{2} / b=a /(1-s)$ with the same magnitude and inverted direction. Up to now, the hub vortex is excluded from the considerations. Considering the hub vortex as visualized in Figure 4, bottom right yields for the complex potential

$$
F(\zeta)=-\frac{\mathrm{i} \Gamma}{2 \pi}\left[\ln (\zeta-b)-\ln \left(\zeta-\frac{a^{2}}{b}\right)\right]+\text { const. }
$$

In the following, this analysis shows that an induced movement of the gap vortex occurs against the rotating direction of the turbomachine at heavy overload. A potential vortex induces a velocity on the surrounding flow. The velocity components of a given potential $F(\zeta)$ are calculated by

$$
\frac{\mathrm{d} F(\zeta)}{\mathrm{d} \zeta}=u_{\text {ind }} \vec{e}_{\zeta}-\mathrm{i} v_{\text {ind }} \vec{e}_{\eta}
$$

A straight vortex filament does not induce a velocity on its own due to the Biot-Savart law so the induced velocity at $\zeta=b$ is only due to the mirrored tip vortex at $\zeta=a /(1-s)$. The resulting induced velocity at the position of the tip vortex yields

$$
\begin{aligned}
u_{\text {ind }} \vec{e}_{\xi}-\mathrm{i} v_{\text {ind }} \vec{e}_{\eta} & =\left.\frac{\mathrm{d}}{\mathrm{d} \zeta}\left[\frac{\mathrm{i} \Gamma}{2 \pi} \ln \left(\zeta-\frac{a^{2}}{b}\right)\right]\right|_{\zeta=b} \\
& =-\frac{\mathrm{i} \Gamma}{2 \pi b} \frac{b^{2}}{a^{2}-b^{2}} \vec{e}_{\eta} .
\end{aligned}
$$

Assuming a turbomachine with $\mathrm{Z}$ impeller blades, the rotating velocity of the tip vortex is

$$
\begin{aligned}
v_{\text {ind }} & =\frac{Z \Gamma}{2 \pi b} \frac{b^{2}}{a^{2}-b^{2}}=\frac{Z \Gamma}{2 \pi a s} \frac{1-s}{2-s} \\
& =\Omega a \frac{1-\varphi / \hat{\varphi}}{s} \frac{(1-s)^{3}}{2-s} .
\end{aligned}
$$

This is the rotating direction against the rotating direction of the turbomachine. For symmetry reasons, the rotating trajectory defines a circular path at radius $b=a(1-s)$. Hence, the induced frequency at $\zeta=b$ is

$$
\Omega_{\text {ind }}=\frac{v_{\text {ind }}}{b}=\frac{\Omega}{s} \frac{(1-s)^{2}}{2-s}\left(1-\frac{\varphi}{\hat{\varphi}}\right) .
$$

For high flow number $\varphi \rightarrow \hat{\varphi}$ and small gap $s \ll 1$, the induced frequency yields

$$
\frac{\Omega_{\text {ind }}}{\Omega}=\frac{1}{2 s}, \quad \text { for } \varphi \rightarrow \infty
$$

against the rotating direction of the turbomachine.

\section{Application for Acoustical Investigations}

Previous investigations by Karstadt et al. [4] analyzed the tip clearance noise in axial turbomachines. Figure 5 shows the frequency spectra over the complete operating range. Remarkable are the peaks at $42 \mathrm{~Hz}$ and $375 \mathrm{~Hz}$, which correspond to the rotational speed $n$ and the blade passing frequency $\mathrm{Zn}$. Fukano and Yang [16] showed that the circumferential frequency of the tip clearance noise shifts to lower values with increasing tip clearance $s$ and decreasing flow number $\varphi$ due to the larger extent of the gap vortex. The present paper investigates the frequency of the tip clearance noise depending on the operating point applying analytical methods. At heavy part load, Equation (2) indicates that an induced frequency at half the rotation speed of the turbomachine should appear. This frequency was also observed by Karstadt et al. [4] as Figure 5 shows a high intensity in the region 
of $0-50 \mathrm{~Hz}$. Equation (17) indicates that for heavy overload the induced frequency will increase with decreasing tip clearance. Furthermore, we expect a noise of high frequency due to the small value of $s<1 \%$ which is common for turbomachines. The broadband drop in the sound power for all flow numbers is clearly visible.

Müller [23] applied the continuity and the momentum equation and deduced that sound inside a fluid volume is only emitted if the rotation of the velocity field changes in time. The present study applies a similar approach to analyze the tip clearance noise of a turbomachine. Time-consuming simulations as performed by Carolus et al. [24] surely allow a more profound and accurate insight into the acoustics of turbomachines. The development of an analytical model which predicts main frequencies is yet interesting to generate a deeper understanding of the acoustics in turbomachines.

These findings and the presented analytical model in this paper could be an efficient tool for acoustic design of turbomachines. This is because this analysis separates kinematic and dynamic effects. Up to now, this is not possible for computational fluid dynamics (CFD) calculations. Separating different effects generates a deeper understanding of the underlying physics and allows realizing particularly focused investigations.

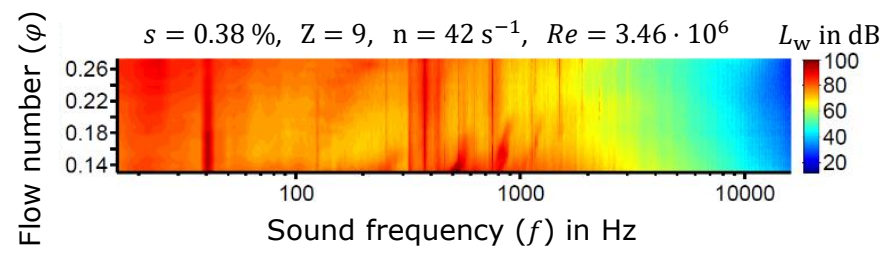

Figure 5. Frequency spectra of the tip clearance noise depending on the flow number $\varphi[4]$.

\section{Summary and Conclusions}

An interplay between dynamic and kinematic effects explains flow structures and phenomena. Using computational fluid dynamics, a clear distinction of both effects is often impossible. In contrast, analytical methods allow a more focused picture of fluid mechanics, i.e., they allow a clear distinction of effects. Of course, only generic flows are accessible to analytical methods.

This paper focuses on an analytic model for wall stall so far being explained by dynamics only: boundary layer separation is indeed a dynamic effect. Nevertheless, boundary layer separation is not necessarily the only reason for wall stall. It is shown that kinematics may also explain at least some effects of wall and rotating stall. The used picture for a flow at small flow numbers is a thread spool rolling up the tip vortices resulting from rotating bound vortices. From the fluid mechanics perspective, the thread spool is a coaxial vortex ring of increasing strength connected to a semi-infinite hub vortex (Figure 1).

Thus far, the velocity potential of a coaxial vortex ring inside a tube was unknown. The solution of Laplace's equation results in the velocity potential for the vortex filament within a tube (see Equation (4)).

This study gained three main results, which are due to kinematics only. First, at part load operation the hub vortex induces a sub-synchronous rotation of the vortex ring. The derived rotational speed $\Omega_{\text {ind }}=0.5 \Omega$ of the vortex ring is surprisingly consistent with observed sub-synchronous speeds of rotating stall (cf. [10]). Second, the vortex ring induces an upstream axial velocity at the wall. Together with the undisturbed flow velocity, this results in a stagnation point upstream and downstream at the wall which may be interpreted as wall stall (Figure 3). Third, at overload operation, the induced rotational direction is inverted to the case at part load. The semi-infinite straight vortex filament at the outer blade end rotates against the rotating direction of the turbomachine due to the induction of the hub vortex. The induced frequency yields $\Omega_{\text {ind }}=-0.5 \Omega / \mathrm{s}$. 
The presented analytical model may give new arguments and improves the understanding of the vortex system in turbomachines but is also intended to motivate generic experiments. Hence, a test rig will validate the models presented in this paper in the near future.

As a next step, the velocity potential for a coaxial vortex ring filament in a circular tube (Equation (4)) will be extended to a coaxial vortex layer, yielding a transient behavior of the vortex system. This behavior leads to a change in the circulation over time being responsible for noise emission [23].

Author Contributions: P.F.P. developed the analytical model. P.T. and F.-J.C. performed the calculations. P.F.P. and P.T. analyzed the results and wrote the paper.

Conflicts of Interest: The authors declare no conflict of interest.

\section{Nomenclature}

\section{Quantity Description}

a tube radius

$b \quad$ blade tip radius

F complex potential

$\Delta h_{t} \quad$ total enthalpy difference

i imaginary number

$J_{n}$

$k_{n}$

$\dot{m}$

$M$

$n$

$P$

$r$

$s$

$t$

$u, v$

$u_{\text {ind }}, v_{\text {ind }}$

$U$

$z$

mass flux

torque

rotational speed

power

radial coordinate

time

velocity

induced velocity

free-stream velocity

axial coordinate

wall stall location

blade number

trailing edge angle

vortex strength

optimal flow number

maximum flow number

velocity potential

Stokes stream function

frequency

induced frequency
Bessel function of order $n, J_{n}(x)=\pi^{-1} \int_{0}^{\pi} \cos (n \tau-x \sin \tau) \mathrm{d} \tau$ $n$-th zero of Bessel function $J_{1}$

tip clearance, $s=(a-b) / a$

dimensionless vortex ring radius, $\beta=b / a$

time-dependent vortex strength, $\Gamma_{t}=Z \Gamma n t$

complex coordinate, $\zeta=\xi+\mathrm{i} \eta$

real part of complex coordinate $\zeta$

imaginary part of complex coordinate $\zeta$

argument of complex coordinate $\zeta$

dimensionless vortex strength, $\tau=\Gamma_{t} /(2 b U)$

flow number, $\varphi=U /(\Omega a)$

dimensionless velocity potential, $\phi=\Phi /(U a)$

dimensionless stream function, $\psi=\Psi /\left(U a^{2}\right)$

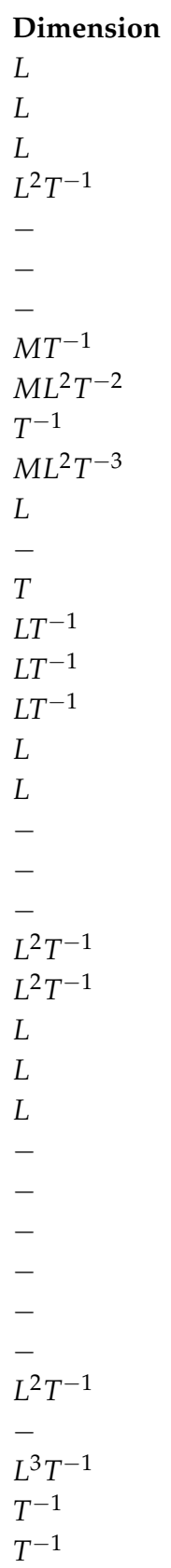




\section{Appendix A. Euler's Turbine Equation}

To obtain $Z \Gamma / 2 \pi=\mathrm{d} M / \mathrm{d} \dot{m}$, the control volume sketched in Figure A1 is considered. The calculation applies the conservation of momentum

$$
\mathrm{d} M=\mathrm{d} \dot{m}\left(r_{2} c_{\mathbf{u} 2}-r_{1} c_{\mathbf{u} 1}\right),
$$

with $c_{\mathrm{u} j}$ the angular component of the total velocity $c$ at position $j$. This equation is also known as Euler's turbine equation. Evaluating the circulation $\Gamma:=\oint_{C} \vec{c} \cdot d \vec{x}$ at a closed curve $C$ yields

$$
\Gamma:=\oint_{C} \vec{c} \cdot \mathrm{d} \vec{x}=\int_{0}^{2 \pi} \vec{c} \cdot \vec{e}_{\varphi} r \mathrm{~d} \varphi=2 \pi r c_{\mathrm{u}} .
$$

Combining Equations (A1) and (A2) and following Helmholtz's theorems, i.e., $\Gamma_{2}-\Gamma_{1}=Z \Gamma$, with the blade number $Z$, the calculation results in

$$
\frac{Z \Gamma}{2 \pi}=\frac{\mathrm{d} M}{\mathrm{~d} \dot{m}} .
$$

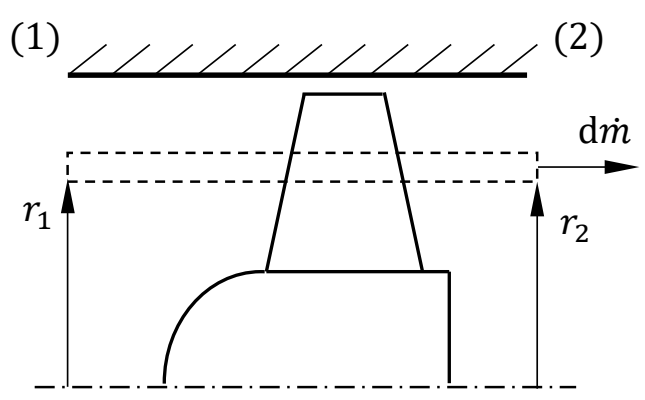

Figure A1. Control volume inside an encased axial turbomachine between the inlet and outlet, Locations (1) and (2), respectively.

\section{Appendix B. Bessel Function}

To obtain the velocity potential for a coaxial vortex ring inside a tube, Equation (4), Laplace's Equation (3) has to be solved for an incompressible, axisymmetric flow. Applying the separation ansatz

$$
\Phi=U z+F(r) G(z)
$$

the velocity potential of the parallel flow $U z$ is superimposed by the potential $F(r) G(z)$ of the vortex ring within the tube. The functions $F(r)$ and $G(z)$ are unknown thus far. To determine $F(r)$ and $G(z)$, an artificial vortex core is assumed first. Second, applying an asymptotic limit, we shrink this vortex core to zero obtaining the singularity solution for a vortex ring filament in a circular tube.

In the first step, we solve the regular boundary value problem sketched in Figure A2.

The vortex core of the annular vortex is smeared to a coaxial, plane washer $b(1-\varepsilon) \leq r \leq b$, with $\varepsilon$ the dimensionless radial extent of the vortex core. For $z=0_{+}$, the vortex induces the radial velocity $W$. For $z=0_{-}$, it induces $-W$ yielding the circulation of the ring

$$
\Gamma_{t}=\oint_{C} \vec{u} \cdot \mathrm{d} \vec{x}=2 \varepsilon b W .
$$

The function $G(z)$ is odd, i.e., $G(-z)=-G(z)$, and vanishes for $G(z \rightarrow \pm \infty)=0$. The boundary conditions for the function $F(r)$ are 


$$
\begin{aligned}
-\infty<z<\infty: & \left.\frac{\mathrm{d} F}{\mathrm{~d} r}\right|_{r=0}=\left.\frac{\mathrm{d} F}{\mathrm{~d} r}\right|_{r=a}=0, \\
z=0: & \frac{\mathrm{d} F(r)}{\mathrm{d} r}= \begin{cases}0, & b<r<a, \\
W, & b(1-\varepsilon)<r \leq b, \\
0, & 0<r \leq b(1-\varepsilon) .\end{cases}
\end{aligned}
$$

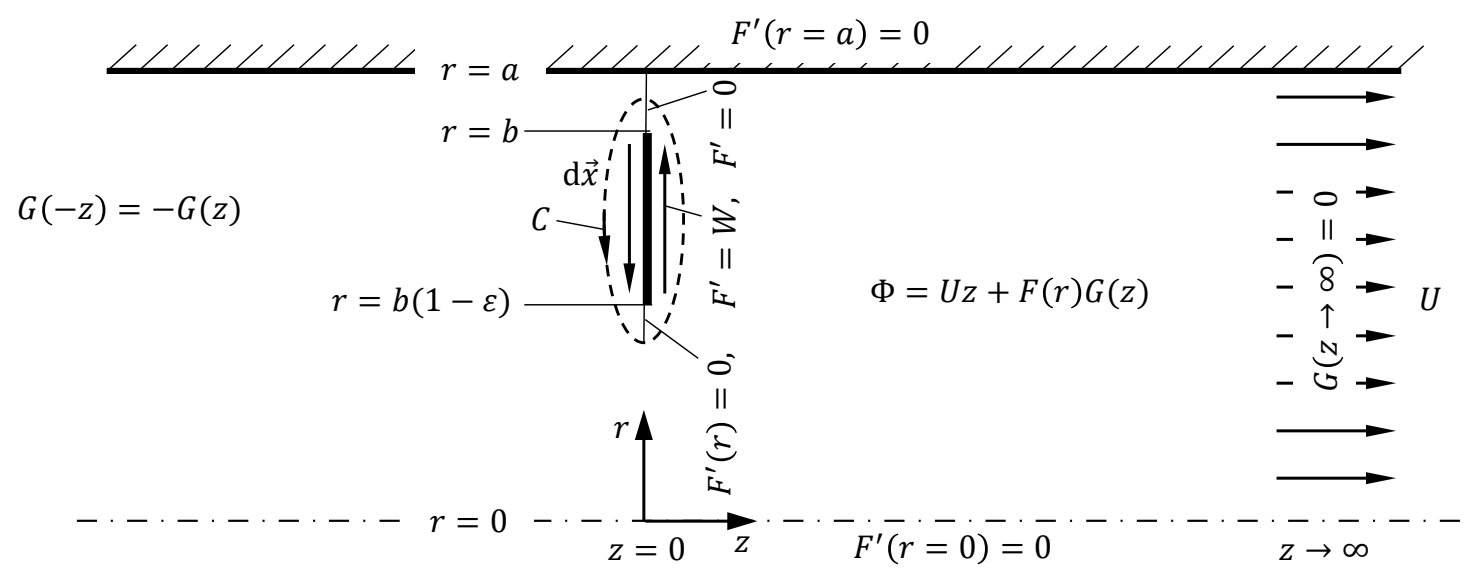

Figure A2. The regular boundary value problem for the vortex ring within a circular tube.

The boundary condition, Equation (A7), is developed in a Bessel series expansion benefiting from the orthogonality properties of the Bessel functions [25]. The solution of Equation (3) satisfying the boundary conditions in Equations (A6) and (A7), yields

$$
\phi_{\varepsilon}(r, z):=\frac{\Phi_{\varepsilon}}{U a}=\frac{z}{a}-\tau \sum_{n=1}^{\infty} \frac{2}{k_{n}} \frac{J_{0}\left(k_{n} r / a\right)}{J_{0}^{2}\left(k_{n}\right)} \exp \left(-k_{n} \frac{|z|}{a}\right) \frac{1}{\varepsilon} \int_{\beta(1-\varepsilon)}^{\beta} J_{1}\left(k_{n} \frac{r}{a}\right) \frac{r}{a} \mathrm{~d}\left(\frac{r}{a}\right),
$$

In the second step, we employ the limit

$$
\phi=\lim _{\varepsilon \rightarrow 0} \phi_{\varepsilon}, \quad \text { with } \Gamma_{t}=2 \varepsilon b W=\text { const },
$$

to gain the asymptotic solution for the vortex filament. Using l'Hôpital's rule, a regular solution for the integral

$$
\lim _{\varepsilon \rightarrow 0} \frac{1}{\varepsilon} \int_{\beta(1-\varepsilon)}^{\beta} J_{1}\left(k_{n} \frac{r}{a}\right) \frac{r}{a} \mathrm{~d}\left(\frac{r}{a}\right)=\beta^{2} J_{1}\left(k_{n} \beta\right)
$$

is found. Hence, the asymptotic limit $\varepsilon \rightarrow 0$ and $\Gamma_{t}=$ const leads to the velocity potential for a coaxial vortex ring filament inside a circular tube

$$
\phi(r, z):=\frac{\Phi}{U a}=\frac{z}{a}-2 \tau \beta^{2} \sum_{n=1}^{\infty} \frac{J_{1}\left(k_{n} \beta\right)}{k_{n} J_{0}^{2}\left(k_{n}\right)} J_{0}\left(k_{n} \frac{r}{a}\right) \exp \left(-k_{n} \frac{|z|}{a}\right) .
$$




\section{References}

1. Greitzer, E.M. Review-Axial Compressor Stall Phenomena. J. Fluids Eng. 1980, 102, 134-151, doi:10.1115/1.3240634. [CrossRef]

2. Dixon, S.L. Some Three Dimensional Effects of Rotating Stall; HM Stationery Office: London, UK, 1962.

3. Cloos, F.J.; Stapp, D.; Pelz, P.F. Swirl boundary layer and flow separation at the inlet of a rotating pipe. J. Fluid Mech. 2017, 811, 350-371, doi:10.1017/jfm.2016.734. [CrossRef]

4. Karstadt, S.; Matyschok, B.; Pelz, P.F. Sound Deadening on Fans. In Proceedings of the ASME Turbo Expo 2011: Turbine Technical Conference and Exposition, Vancouver, BC, Canada, 6-10 June 2011; American Society of Mechanical Engineers: Vancouver, BC, Canada, 2011; pp. 961-971.

5. Zhu, T. On the Flow Induced Tip Clearance Noise in Axial Fans. Ph.D. Thesis, Universität Siegen, Siegen, Germany, 2016.

6. Betz, A. Schraubenpropeller mit geringstem Energieverlust. Mit einem Zusatz von L. Prandtl. In Nachrichten von der Gesellschaft der Wissenschaften zu Göttingen, Mathematisch-Physikalische Klasse; Weidmanndsche Buchhandlung: Göttingen, Germany, 1919; pp. 193-217.

7. Goldstein, S. On the vortex theory of screw propellers. Proc. R. Soc. Lond. Ser. A 1929, 123, 440-465. [CrossRef]

8. Glauert, H. Die Grundlagen der Tragflügel- und Luftschraubentheorie; Springer: Berlin, Germany, 1929.

9. Van Kuik, G. The relationship between loads and power of a rotor and an actuator disc. J. Phys. Conf. Ser. 2014, 555, 012101, doi:10.1088/1742-6596/555/1/012101. [CrossRef]

10. Brennen, C.E. Hydrodynamics of Pumps; Oxford Science Publications, Concepts ETI; Oxford University Press: Norwich, VT, USA; Oxford, UK; New York, NY, USA, 1994.

11. Helmholtz, H. Über Integrale der hydrodynamischen Gleichungen welche den Wirbelbewegungen entsprechen. J. für die Reine und Angewandte Math. 1858, 55, 25-55. [CrossRef]

12. Didden, N. On the formation of vortex rings: rolling-up and production of circulation. Z. Angew. Math. Phys. (ZAMP) 1979, 30, 101-116. [CrossRef]

13. Longhouse, R.E. Control of tip-vortex noise of axial flow fans by rotating shrouds. J. Sound Vib. 1978, 58, 201-214. [CrossRef]

14. Brooks, T.F.; Marcolini, M.A. Airfoil tip vortex formation noise. AIAA J. 1986, 24, 246-252, doi:10.2514/3.9252. [CrossRef]

15. Mailach, R.; Lehmann, I.; Vogeler, K. Rotating Instabilities in an Axial Compressor Originating From the Fluctuating Blade Tip Vortex. J. Turbomach. 2001, 123, 453, doi:10.1115/1.1370160. [CrossRef]

16. Fukano, T.; Jang, C.M. Tip clearance noise of axial flow fans operating at design and off-design condition. J. Sound Vib. 2004, 275, 1027-1050, doi:10.1016/S0022-460X(03)00815-0. [CrossRef]

17. McDougall, N.M.; Cumpsty, N.A.; Hynes, T.P. Stall inception in axial compressors. In Proceedings of the ASME 1989 International Gas Turbine and Aeroengine Congress and Exposition, Toronto, ON, Canada, 4-8 June 1989; American Society of Mechanical Engineers: Toronto, ON, Canada, 1989; pp. 116-125.

18. Saathoff, H.; Stark, U. Tip Clearance Flow Induced Endwall Boundary Layer Separation in a Single-Stage Axial-Flow Low-Speed Compressor. In Proceedings of the ASME Turbo Expo 2000: Turbine Technical Conference and Exposition, Munich, Germany, 8-11 May 2000; ASME: Munich, Germany, 2000, doi:10.1115/2000-GT-0501. [CrossRef]

19. Karstadt, S.; Pelz, P.F. A Physical Model for the Tip Vortex Loss: Experimental Validation and Scaling Method. In Proceedings of the ASME Turbo Expo 2012: Turbine Technical Conference and Exposition, Copenhagen, Denmark, 11-15 June 2012; American Society of Mechanical Engineers: Copenhagen, Denmark, 2012; pp. 73-81.

20. Prandtl, L. Über die Entstehung von Wirbeln in der idealen Flüssigkeit, mit Anwendung auf die Tragflügeltheorie und andere Aufgaben. In Vorträge aus dem Gebiete der Hydro-und Aerodynamik; Springer: Innsbruck, Austria, 1924; pp. 18-33.

21. Saffman, P.G. Vortex Dynamics; Cambridge University Press: Cambridge, UK, 1992.

22. Milne-Thomson, L.M. Theoretical Hydrodynamics; Courier Corporation: New York, NY, USA, 1968.

23. Müller, E.A. Der Wirbel als Schallerzeuger. In Ernst Becker Gedächtnis Kolloquium; 28; Schriftenreihe Wissenschaft und Technik: Darmstadt, Germany, 1985; pp. 137-151. 
24. Carolus, T.; Schneider, M.; Reese, H. Axial flow fan broad-band noise and prediction. J. Sound Vib. 2007, 300, 50-70, doi:10.1016/j.jsv.2006.07.025. [CrossRef]

25. Pelz, P.F.; Spurk, J.H.; Müller, H.D.J. Reducing mixing at the outlet bore of a cylinder. Arch. Appl. Mech. 1998, 68, 395-406. [CrossRef]

(C) 2018 by the authors. Licensee MDPI, Basel, Switzerland. This article is an open access article distributed under the terms and conditions of the Creative Commons Attribution NonCommercial NoDerivatives (CC BY-NC-ND) license (https://creativecommons.org/licenses/by-nc-nd/4.0/). 